Session 2520

\title{
Teaching Electric Circuits via the Internet
}

\author{
J. A. Svoboda \\ Clarkson University
}

\section{Introduction}

The Electronic Teaching Assistant (ETA) is a computer tool designed to aid both in teaching and in learning about electric circuits. The ETA consists of html documents containing java applets and so is ideally suited to the Internet. This paper presents the educational objectives (both teaching and learning) of the ETA and describes how the ETA accomplishes those objectives.

The ETA consists of three parts: the Electric Circuits Workout, the Circuit Design Lab, and the Interactive Illustrations. The Electric Circuits Workout provides an opportunity to develop and practice circuit analysis skills by presenting exercises similar to quiz or exam problems. The Electric Circuits Workout poses a problem, then accepts and checks the user's answer.

The Circuit Design Lab provides an opportunity for experimentation and design. Each lab exercise presents a circuit together with scrollbars that control the values of important circuit parameters. This provides an opportunity for "What if..." explorations as the effects of changing parameter values are observed. The Circuit Design Lab also provides an opportunity for design exercises.

The Interactive Illustrations provide an opportunity to change some aspect of an illustration and observe the consequence. Such illustrations emphasize the connection between concepts. For example, one of the Interactive Illustrations shows a phasor and the corresponding sinusoid. The student interacts with the illustration by changing the phasor using the computer mouse. The Interactive Illustrations responds by changing the sinusoid appropriately, emphasizing the relationship between phasor and sinusoid.

The ETA has been class tested for two years. The goal of the ETA has evolved as a result of that testing. Initially the goal was very simple: to use the Internet to help motivated students learn about circuits. Later the ETA was organized into its three parts by observing which applets had been successful and identifying their common characteristics. In addition to helping students, the ETA saved time for instructors by providing homework problems that are graded automatically, exam problems ready to cut and paste into exams or quizzes and demonstrations ready to be incorporated into lectures. Observing that the ETA benefited instructors as well as students changed the intended audience and, correspondingly, the goals of the ETA. The ETA now has two goals: to reduce the time and effort required of the instructor and to help the students learn about circuits. This is an important change since the ETA is most successful when instructors enthusiastically incorporate it into their course. 


\section{Electric Circuits Workout}

The Electric Circuits Workout is designed to help students learn about electric circuits by providing numerous practice problems and immediate feedback regarding the correctness of the students solutions to those problems. The problems are separated by topic. At present, the Electric Circuits Workout includes problems on 16 topics:

1. Ohm's and Kirchhoff's Laws: Simple circuits

2. Ohm's and Kirchhoff's Laws: Circuits with Dependent Sources

3. Voltage and Current Division, Series and Parallel Resistors

4. Equivalent Resistance

5. Mesh and Node Equations: Simple Circuits

6. Mesh and Node Equations: More Complex Circuits

7. Mesh and Node Equations: Circuits with Dependent Sources

8. Superposition

9. Thevenin and Norton Equivalent Circuits

10. Operational Amplifier Circuits: Inverting and Noninverting Amplifiers

11. Capacitors and Inductors

12. Complete Response of Simple 1st Order Circuits

13. Complete Response of 1st Order Circuits

14. Voltage Division for AC Circuits

15. KCL and KVL for AC Circuits

16. AC Power

The workout for each topic presents circuits having several circuit topologies and randomly chosen parameter values. Figure 1 shows a screen from the Electric Circuits Workout.

\section{Electric Circuits Workout}

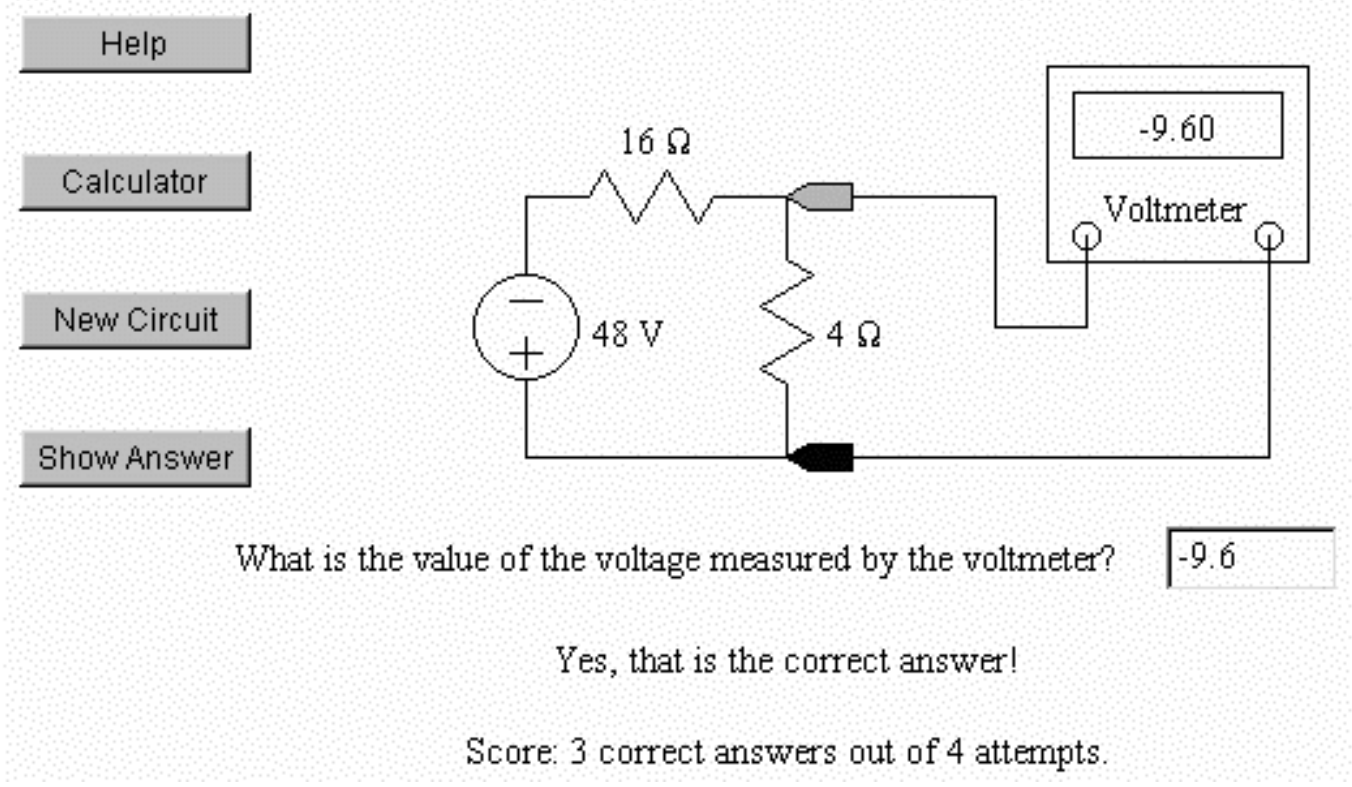

Figure 1 A screen from the Electric Circuits Workout 
Figure 1 shows several lines of text below the drawing of the circuit. In the first line of text, the Electric Circuits Workout asks a question and provides a place for the answer. The second line provides feedback by indicating whether or not the answer is correct. The third line displays a score of the form "x correct answers out of y attempts."

The buttons at the left of the screen shown in Figure 1 show how the student uses Electric Circuits Workout. The top button provides a help screen. A separate help screen is provided for each topic. This help screen identifies the principles needed to solve the problems in this topic and also provides a reference to an explanation of the relevant theory [7]. Frequently, that explanation includes solutions of the very problems presented by Electric Circuits Workout, albeit with different parameter values.

The second button from the top pops up a calculator. The third button from the top causes the Electric Circuits Workout to generate a new problem. The fourth button causes the Electric Circuits Workout to display the correct answer. The "Show Answer" button is a toggle button, so pressing it a second time hides the answer. Of course, the Electric Circuits Workout will not accept an answer from the user after showing the user the correct answer.

The objective of the Electric Circuits Workout is to provide a set of homework problems that are graded automatically and immediately. The Electric Circuits Workout is a modern version of an earlier program called the Linear Circuits Tutor [1,2,3,4,5]. The Linear Circuits Tutor also provided automatically graded homework problems. The author's experience using the Linear Circuits Tutor guided design of the Electric Circuits Workout. In particular, the pop-up calculator and the "Show Answer" feature respond to student comments regarding the perceived shortcomings of the Linear Circuits Tutor. Similarly, the help screens were added in response to student comments regarding an early version of the Electric Circuits Workout. The current version of the Electric Circuits Workout has been used in a sophomore course on electric circuits for two years and has been well received by the students in those classes. Indeed, many students report spending most of their homework time using the Electric Circuits Workout, sometimes to the detriment of other homework assigned in the circuits course. Hence, it is important to identify good problems for the Electric Circuits Workout.

Two principles guide the design of problems for the Electric Circuits Workout. First, the answer must be a real number. This rule is not as restrictive as it may first seem. The real number could represent the amplitude of a sinusoid, the time constant of a first order circuit or a particular coefficient in a partial fraction expansion. Second, the problem should ask the student to perform a single calculation rather than a series of calculations. Recall that the feedback provided by the Electric Circuits Workout indicates whether an answer is correct or not. This is adequate feedback when the answer is obtained by writing and solving a single equation, but not when a series of calculations must be performed. This second rule is also not overly restrictive since students can be led through a series of calculations by asking for a series of questions. The Electric Circuits Workout checks the answer to each before asking the next question. Further, The Electric Circuits Workout displays the answer to the first question while asking the second question. 
It is a simple matter to incorporate problems from the Electric Circuits Workout into quizzes or exams. Graphic editing tools available as freeware can be used to "cut and paste" problems from the Electric Circuits Workout into quiz or exam documents prepared using popular word processors. (Step-by-step instructions are posted on the author's web site [6].) Both instructors and students benefit from using problems from the Electric Circuits Workout in exams and quizzes. Instructors benefit from reduced exam preparation time. The Electric Circuits Workout provides not only the problem but also the correct answer. The error checking built into the Electric Circuits Workout reduces the error checking needed to write the exam. Further, the Electric Circuits Workout will produce several versions of a problem, each with different parameter values, and each with the correct answer. This feature saves a lot of time when multiple versions of an exam must be prepared. Students appreciate the fairness of incorporating problems into exams that are similar to the problems that they have practiced.

The Electric Circuits Workout has also be used to collect homework grades remotely using the Internet. Remote grading requires a java severlet to receive and record the grades and also a modified applet that can send grades to the servlet. The "Voltage Division for AC Circuits" applet was modified by adding a "Submit" button. In response to the "Submit" button, the modified applet collected some information about the student, e.g. name and student number, and sent this information and the number of problems worked correctly to the servlet. The servlet and modified applet were successfully used to collect homework grades for a class of 250 students.

When the opportunity presents itself, the Electric Circuits Workout provides more information that is needed to solve a problem. Consequently, the student must select the information that will be used, rather than looking for a formula that uses all the available information.

\section{Circuit Design Lab}

The Circuit Design Lab provides an opportunity to experiment with particular circuits by adjusting values of parameters and observing the results. Each Design Lab presents a circuit together with scrollbars for adjusting the value of the circuit parameters. Figure 2 shows a screen from the Circuits Design Lab. A variety of circuits are included in the Circuit Design Lab:

$\begin{array}{llll}\text { 1. } & \text { VCVS Circuit } & 10 . & \text { Amplifier using VCVS } \\ \text { 2. } & \text { VCCS Circuit } & 11 . & \text { Inverting Amplifier } \\ \text { 3. } & \text { CCVS Circuit } & 12 . & \text { Noninverting Amplifier } \\ \text { 4. } & \text { CCCS Circuit } & 13 . & \text { RL Circuit: Transient Response } \\ \text { 5. } & \text { Voltage Divider } & 14 . & \text { RC Circuit: Transient Response } \\ \text { 6. } & \text { Current Divider } & 15 . & \text { RL Circuit: AC Response } \\ \text { 7. } & \text { Series Resistors } & 16 . & \text { RC Circuit: AC Response } \\ \text { 8. } & \text { Parallel Resistors } & 17 . & \text { Coupled Coils Circuit } \\ \text { 9. } & \text { Equivalent Resistance } & 18 . & \text { Ideal Transformer Circuit }\end{array}$

Each Circuit Design Lab consists of a java applet embedded in an html page. The java applet provides interactivity while the html page provides a context. This context has two parts: a discussion of the equations used to design the circuit and a graduated set of design problems. The 
set of design problem starts with very simple problems. The problems gradually increase in difficulty until, finally, students are asked to design the circuit to satisfy typical specifications.

Two things, an applet tag in the html page and a zip file, are required to make an applet work. Hence it is easy for instructors to create a new context for the applet by moving it to their own html page, with appropriate attribution, of course. Only two steps are required. First, copy the entire applet tag, verbatim, to the new html page. Second, copy the zip file into the same directory (folder) as the new html file. (More detailed instructions are available on the author's web site [6]. These instructions include a template for the new html file that already includes the applet tag.)

The Circuit Design Lab has been used successfully in two ways. First, in a lecture hall equipped with appropriate projection facilities, instructors can demonstrate the operation and design of a circuit using the Circuit Design Lab. Second, the Circuit Design Lab, can be used as homework to prepare for a quiz that asks to design the circuit to satisfy typical specifications. For example, the Circuit Design Lab on voltage dividers prepares students for a quiz that asks them to "design a voltage divider having a gain of $0.9 \mathrm{~V} / \mathrm{V}$." In contrast, merely telling students that the Circuit Design Lab is available and hoping that they will use it voluntarily has not been successful.
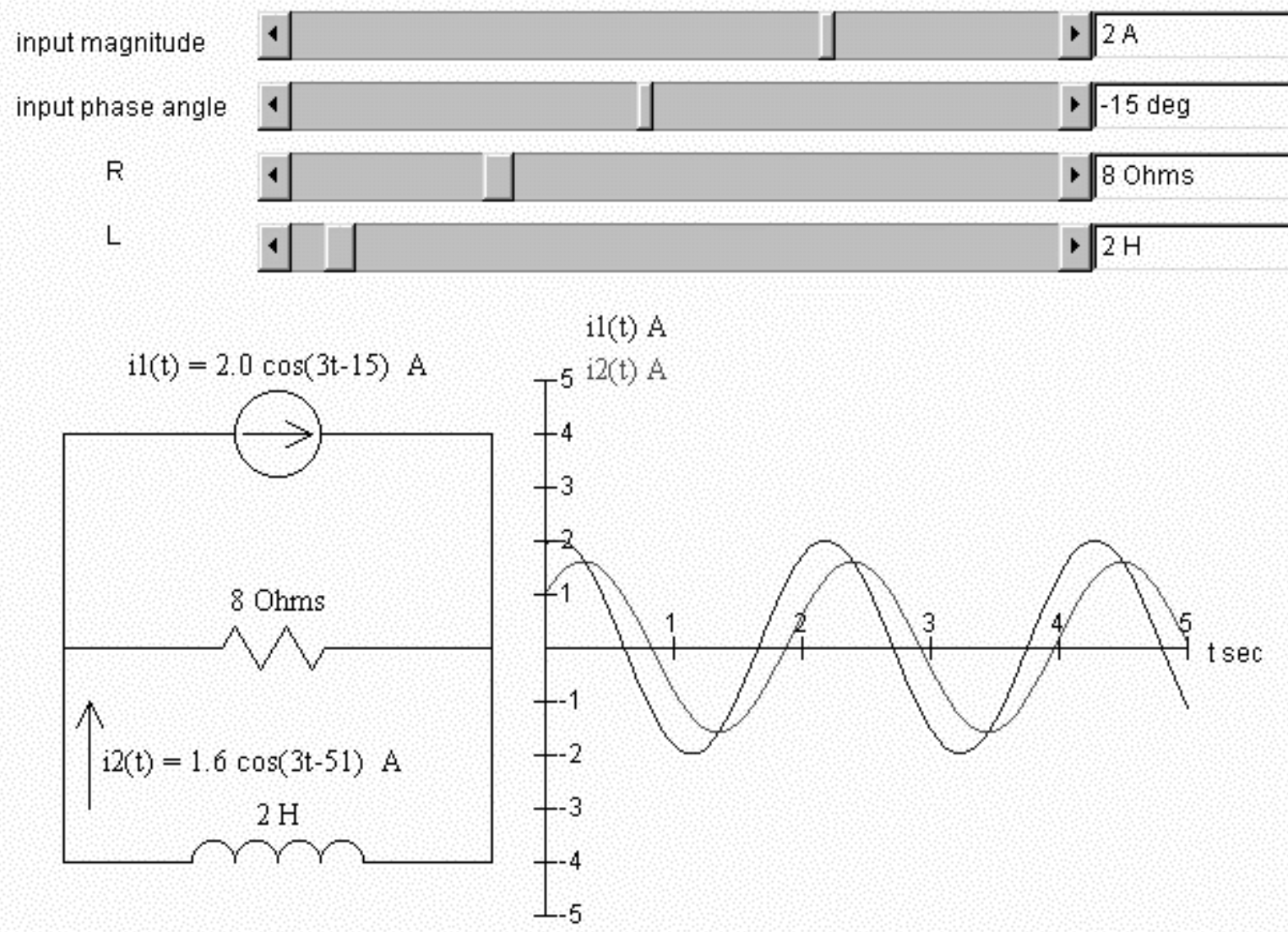

Figure 2 A screen from the Circuits Design Lab. The plots are distinguished using color, that is, both the label and trace for i1(t) are blue while both the label and trace for i1(t) are red. 


\section{Interactive Illustrations}

Modern software provides the tools needed to create a Graphical User Interface (GUI). The user interacts with the GUI using mouse clicks as well as key strokes. The Interactive Illustrations use the Graphical User Interface (GUI) to draw the user's attention to connections between related concepts. For example, consider Figure 3, which shows a screen from one of the Interactive Illustrations. This illustration is used to describe the connection between a phasor in the frequency domain and the corresponding sinusoid in the time domain [7]. The applet displays an illustration and the user is asked to interact with the illustration, in this case through mouse clicks. To be specific, the user is asked to specify a new phasor by clicking the mouse in the square identified as the frequency domain. The user's mouse click specifies a new phasor and the applet responds by changing the sinusoid to match the phasor. The user changes the phasor using a mouse click and the applet changes the sinusoid. These steps can be repeated several times, until the connection between the phasor and the sinusoid becomes clear.

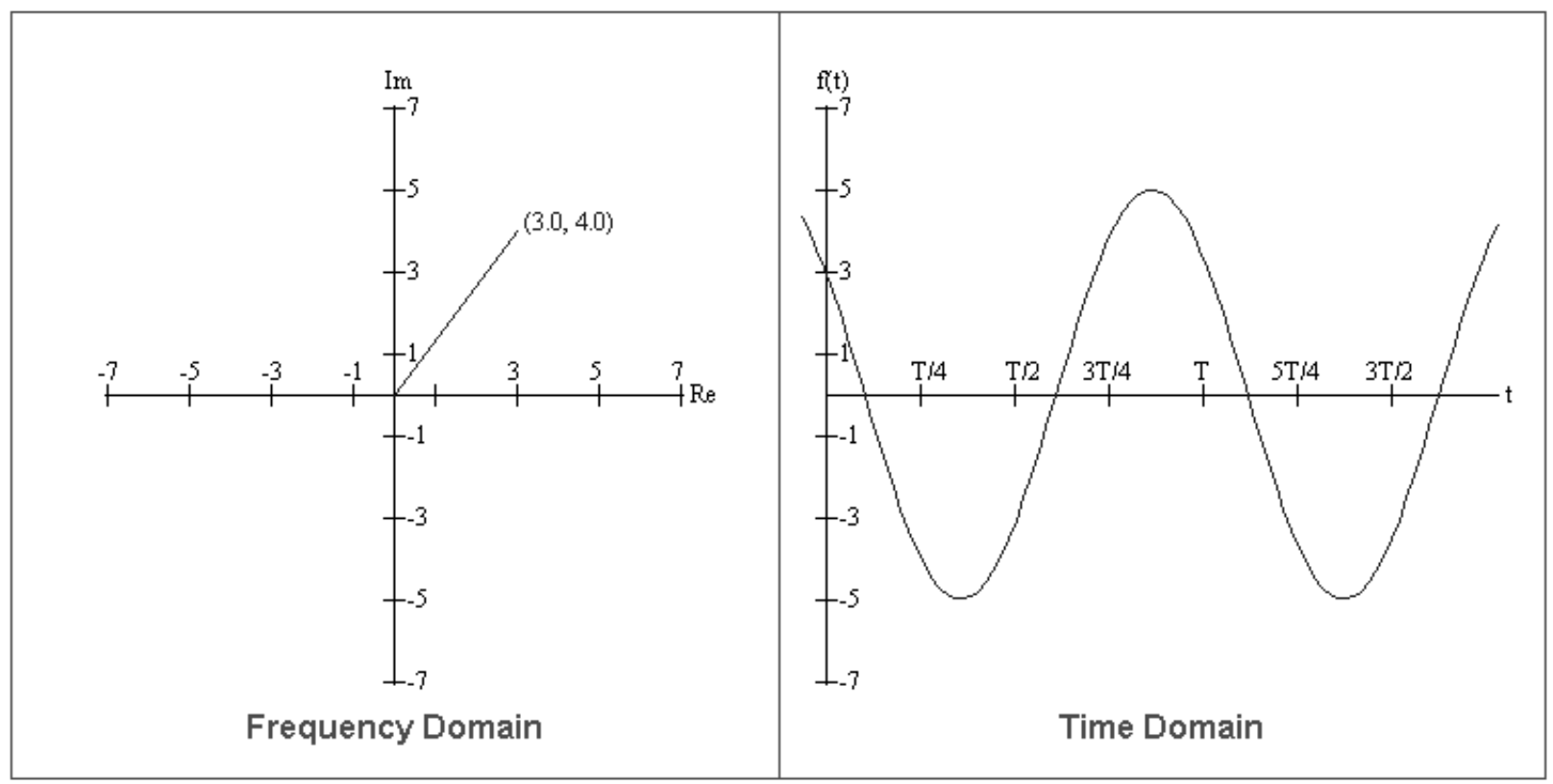

Figure 3 A screen from the Interactive Illustrations.

Each of the applets in the Interactive Illustrations follows the same pattern. First, the applet illustration is displayed. Next, the user interacts with the illustration, usually through mouse clicks. The user's interaction changes the illustration. Finally, the applet responds by making a corresponding change to the illustration. The user's interaction draws his attention to the change he made and the corresponding change made by the applet.

The Interactive Illustrations are a miscellaneous set of applets. Each was developed when it seemed that a class needed help making a connection between related concepts. Interactive Illustrations are provided for the following topics: 
1. Voltage and Current Reference Directions

2. Thevenin and Norton Equivalent Circuits

3. Superposition

4. Response of First Order Circuits

5. Phasors and Sinusoids

6. Matching Phasors to Sinusoids

7. Adding Phasors

The Interactive Illustrations have been used successfully in two ways. First, in a lecture hall equipped with appropriate projection facilities, instructors can incorporate the Interactive Illustrations into their lectures. Second, the Interactive Illustrations can be used during office hours. As with the Circuit Design Lab, merely telling students that the Interactive Illustrations are available and hoping that they will use them voluntarily has not been successful.

\section{Conclusion}

The Electronic Teaching Assistant (ETA) is a computer tool designed to aid both in teaching and in learning about electric circuits. The ETA aids instructors by providing homework, exam problems and lecture material. The ETA aids instructors who use the Internet by providing applets that can be used in the instructors web pages. The ETA aids students by providing homework that is marked immediately. Students also benefit from the opportunity to experiment with the circuits included in the Circuit Design Lab.

\section{References}

[1] Svoboda, J.A. Introducing the Personal Computer in Introductory Electrical Engineering Courses. 1987 ASEE Annual Conference Proceedings. pp.699-705. Reprinted in the Journal of Computers in Education (CoED), vol. 8, no. 4, pp. 1-7, 1988.

[2] Svoboda, J.A. “On the Role of the Personal Computer in Teaching Design”, 1988 ASEE Annual Conference Proceedings , pp.1844-1851. Reprinted in the Journal of Computers in Education (CoED), vol. 9, no. 2, pp. 53-60, 1989.

[3] Svoboda, J.A. On Using the Personal Computer in Teaching Sophomore Circuits. International Journal of Applied Engineering Education, vol. 4, no. 5, pp.387-394, 1988

[4] Demerdash, N.A.O, Gallager, R.H., Schilling, R.J. \& Svoboda, J.A. Impact of Academic Computing on Teaching Electrical Engineering at Clarkson University. IEEE Transactions on Education, vol. 36, no. 1, pp. 94102, February 1993.

[5] Huelsman, L.P. Personal Computer Programs for Electrical Engineering. IEEE Circuits and Devices Magazine. vol. 6, no. 1, pp.3-6, January 1990.

[6] URL: http://www.clarkson.edu/ svoboda/eta/index.html.

[7] Dorf, R.C. \& Svoboda, J.A. Introduction to Electric Circuits: $5^{\text {th }}$ Edition. New York: Wiley (2001). 